\title{
Wanting or having children predicts age preferences in online dating
}

\author{
Robin S. S. Kramer \\ University of Lincoln
}

Alex L. Jones

Swansea University

Correspondence concerning this article should be addressed to Robin S. S. Kramer, School of Psychology, University of Lincoln, Brayford Pool, Lincoln LN6 7TS, United Kingdom. E-mail: remarknibor@gmail.com

\section{Acknowledgments}

The authors thank Abi Davis for critical comments on the manuscript. 


\begin{abstract}
When dating, women seek men slightly older than themselves while men typically prefer younger women. Such patterns reflect differences in parental investment and age-related fertility, which are both concerned with maximising reproductive outcomes. Using large samples of online daters, we considered whether having or wanting children was associated with the perceived importance of age as a matching criterion when dating (Study $1 ; N=119,361$ ), as well as how these two factors related to the preferred age of a match (Study 2; $N=486,382$ ). Men without children (or those who wanted children) rated age as more important than those with children (or those who did not want children), and also selected a preferred age range that incorporated younger women. In contrast, women's preferences showed little association with having or wanting children. Taken together, these findings demonstrate that age preferences may depend on factors in addition to those previously investigated, and that the relationships with the number of current children and the desire to have children are consistent with evolutionary predictions.
\end{abstract}

Keywords: age preferences, mate selection, parental investment, having children, wanting children

\title{
Public Significance Statement
}

"We investigated behaviours in large samples of online daters, considering whether preferred partner age was related to wanting or having children. Although women's preferences showed little association with these factors, men without children (or those who wanted children) rated age as more important than those with children (or those who did not want children), and also selected a preferred age range that incorporated younger women. Our findings extend current theories of mate selection by highlighting the importance of wanting or having children when deciding who we choose to date." 
Evolutionary accounts of human mate choice argue that men and women should value different qualities in potential partners because they are motivated by disparate considerations. This is a result of differential parental investment across the two sexes (Trivers, 1972). While both men and women invest heavily in their offspring, these investments take the form of different resource provisions. Women contribute relatively more of their own physical resources, through both pregnancy and breastfeeding, and so a woman's health and reproductive potential (fecundity and fertility) should be highly valued by men. In contrast, men's contributions are more indirect (food, security, etc.), suggesting that women should place a higher value on the potential for men to provide these types of resources since they themselves may be unable to, being constrained by physical resource provision. In both cases, evidence appears to support these differential motivations (Buss, 1989; Sprecher et al., 1994; Waynforth \& Dunbar, 1995).

By valuing different qualities in a potential mate, men and women are expected to display mating behaviours that reveal different ideal ages for their partners. Although women's highest residual reproductive value (i.e., the most future potential to conceive and invest in children) peaks at menarche (about 12.5 years of age; McDowell et al., 2007), a proportion of their cycles are anovulatory for several years afterwards (Apter, 1980). In addition, chromosomal errors in the eggs of young and older women (Gruhn et al., 2019) mean that peak fertility is reached in women's midtwenties (Dunson et al., 2002; Hawkes \& Smith, 2010; Wood, 1989). Seemingly then, men have therefore evolved to find women in their early to mid-twenties the most sexually attractive, irrespective of their own age, as this has resulted in the greatest inclusive fitness. In contrast, men have the potential to increasingly accumulate economic resources and social status throughout their lives, although physical health and virility (as well as remaining lifespan) will decrease in later years. This trade-off between greater resources in older men and decreasing physical potential, combined with the knowledge that men mature later than women (Daly \& Wilson, 1983; Marshall $\&$ Tanner, 1969, 1970), argue for a female attraction towards men who are older than themselves 
but not significantly so. Again, this behaviour has resulted in the greatest inclusive fitness in their evolutionary past.

At this stage, it is important to consider the distinction between proximate and ultimate explanations for these behaviours (Mayr, 1963; Tinbergen, 1963). The ultimate explanation (typically, the "why" question) can be couched in terms of greater inclusive fitness. For men, a preference for younger women is expected to result in a larger number of subsequent offspring. For women, preferring older men should increase the likelihood that the offspring they produce will survive via resource availability. The proximate explanation (the "how" question) concerns, more directly, this preference for younger or older mates. Specifically, our attraction to age-related characteristics drives our sexual interests and, as a result, our mating decisions, through our evolved psychology.

The predictions regarding differential patterns of age preferences described above are generally borne out in the data (for reviews, see Buss \& Schmitt, 2019, and Conroy-Beam \& Buss, 2019). Evidence for such patterns has been found in both reported sexual interest and activity (Antfolk, 2017; Antfolk et al., 2015), personal advertisements and marriage statistics (Kenrick \& Keefe, 1992; Waynforth \& Dunbar, 1995), online dating profiles (Conway et al., 2015; Dunn et al., 2010; Gustavsson et al., 2008; Phua et al., 2018; Skopek et al., 2011), the purchasing of brides from developing countries (Sohn, 2017), and even in the prices that men are willing to pay for prostitutes (Sohn, 2016). However, preferences in long-term mating may reflect a desire for a partner closer to one's own age (Buunk et al., 2001; Phua et al., 2018), perhaps increasing the likelihood of bonding and cooperation, with the result that parental effort is maximised (Kenrick \& Keefe, 1992).

If partner age preferences reflect evolved drives originating from differential parental investment, it follows that people who already have and/or do not want children may display different preferences. For instance, a man in his forties with children from a previous marriage, who does not want more children, could be less motivated by - at the proximate level - attraction to the supposedly optimal fertility of a woman in her mid-twenties. Instead, a woman closer to his own 
age would be a more suitable partner in terms of indirect resource contribution and compatibility (Kenrick \& Keefe, 1992).

Although theoretically influential, few researchers have considered whether having or wanting children affects age preferences in a partner. In a cross-cultural survey, the number of children desired by men positively correlated with their preferred spousal age difference (Buss et al., 2000). In other words, desiring more children was associated with searching for an even younger woman, who could therefore bear a larger number of future offspring. An online survey of 2,271 people found that men with children reported a weaker preference for younger women, while women with children increasingly preferred same-aged or older men (Skopek et al., 2011). In addition, an analysis of 404 online daters in the Dominican Republic found that users were unaffected by currently having children when specifying their preferred partner's age but those who wanted children sought partners around three years younger than those who did not (Phua et al., 2018). However, the researchers did not consider the possibility of sex differences in how wanting children might relate to age preferences, and the inclusion of daters up to the age of 73 meant that older participants (very few of whom presumably wanted children) will have influenced the resulting regression model's slope, even after controlling for daters' ages.

To investigate whether having or wanting children is associated with age preferences in a partner, we analysed two large datasets provided by eharmony UK, a popular online dating website. We considered the relationship between these factors and age preferences for both men and women, across the full age range of daters. In Study 1, we investigated how important users considered age to be as a matching criterion when dating. Following the argument outlined above, we predicted that users who already had children, or did not want any (more), would judge age to be of less importance when searching for a match. In Study 2, we analysed the minimum and maximum age boundaries specified by a different sample of users. Along similar lines, we predicted that those users who already had or did not want children would respond in ways that reflected this. For men, this might incorporate the fact that female youth and fertility - signals of reproductive capacity 
(Buss, 1989) - were no longer as important since, at the ultimate level, producing offspring was not the goal. Therefore, we predicted that those with children (or who did not want children) would increase their minimum and maximum age preferences to reflect a weaker draw towards women in their 20s (Buss, 1989), who are at their most fertile. For women, predictions were less clear since the desire to have children with a partner could result in the pursuit of men with increased resource potential (Buss, 1989; Buss \& Schmitt, 1993), either in the form of younger men who have a greater remaining lifespan, or older, more mature men who have already accrued resources and social standing but may see decreases in both their health and rate of copulation (Kenrick \& Keefe, 1992). Given that the current literature does not speak to which of these may dominate as wanting or having children is altered, and hence whether the draw operates upwards (towards increasingly older men with resources) or downwards (towards younger and healthier men, and the women's own ages), the current work served to investigate this open question.

In both studies, we also considered how the user's age might interact with these preferences. For men without children (or those who want children), the potential draw towards women in their 20s may result in different preferences across the lifespan. Maximum and minimum preferred ages could show a larger decrease for older men since there is further to fall in order to more closely incorporate women in this category, while younger men would only need to show a smaller decrease, even if drawn to the same category of women. This logic also applies to women without children, although it is unclear as to whether women will prefer increasingly older men or ones nearer their own age, and if this differs across the age range. To address this, we investigated how user gender, age, and having or wanting children might interact when predicting preferences.

\section{Study 1}

In this study, we investigated whether having or wanting children predicted how important users considered age to be as a matching criterion when dating. 


\section{Method}

\section{Dataset}

Profile information was obtained through a collaboration with eharmony UK, who stipulated that the user data analysed here and in Study 2 cannot be made freely available. The dataset comprised information taken from 149,440 website profiles which had previously been used to explore dating preferences and messaging behaviours (Dinh et al., 2018). In all cases, users were living in the UK, heterosexual, and not currently in a relationship. For each profile, the user had self-reported (upon registration) their age, ethnicity, and gender. Importantly for the current study, users reported the number of children they had and whether they wished to have (more) children (yes/maybe/no). Finally, users also rated the importance of age as a matching criterion using a 1 (not very important) to 7 (very important) Likert scale.

From this initial sample, we chose to exclude users who reported having more than seven children since these responses were considered to be false. (Users above this cut-off were infrequent and many of these reported extreme values.) We also chose to include only users aged at least 20 years old for two reasons. First, the site only allowed people to register as users if they were aged 18 and above. As such, we suspected that potential underage users might input their dates of birth (on which this age check is carried out) to imply they matched this minimum age requirement. By setting our minimum to 20 , we would avoid such profiles and their false information. Second, this lower age restriction was necessary in Study 2 and so applying it here made for a more comparable sample from which to make generalisations across the two studies.

We also chose to include only users aged 50 and below for two reasons: 1) the majority of the site's users are below this age and so including older profiles would not necessarily allow us to generalise our findings to older daters; and 2) our main focus is on the association with having and 
wanting children, and the latter is no longer an option for postmenopausal women, which typically occurs around 50 years of age. For example, the average age for a woman in the UK to reach the menopause is 51 (National Health Service, 2021).

Our final sample comprised 119,361 users (50.53\% women; $82.66 \%$ White; age $M=34.80$ years, $S D=7.99$ years). Research ethics for both studies presented here were approved by the university's research ethics committee (ID 3746).

\section{Statistics}

Given that the values in our dataset were necessarily bounded in terms of both age range (2050) and rated importance of age as a matching criterion (1-7), and in line with previous research (Conway et al., 2015), we tested for heteroskedasticity (Breusch \& Pagan, 1979) and found evidence of it in our sample, $\chi^{2}(1)=854.90, p<.001$. Even with significant heteroskedasticity, linear regressions with large samples often result in good performance (Lumley et al., 2002). However, following statistical recommendations and previous work (Conway et al., 2015), we chose to report robust standard errors for all analyses, which adjust for heteroskedasticity, and fit our data using robust linear models.

Through inspection of both probability-probability and quantile-quantile plots, we observed deviations from multivariate normality in our data. There may be several reasons for this, such as our exclusion of users outside of the 20-50 age range. However, for large data sets, a linear regression approach is valid for any distribution as a result of the Central Limit Theorem (Lumley et al., 2002), and as such, we utilised this method of analysis here.

\section{Results}


While theory suggests that both having children and wanting children may predict how users consider the importance of age as a matching criterion, these two factors show substantial overlap in the data. In many cases, users who already had children did not want any more, while those who did not have children expressed a desire to have some in the future (see Table S1). Indeed, a oneway between subjects analysis of variance (ANOVA) found significant differences between groups (wanting children: yes/maybe/no) in terms of the number of children that users already had, $F(2$, $117836)=8843, p<.001, \eta^{2}=0.13$. In all cases, paired comparisons were statistically significant (all $p$ s $<.001$; yes $M=0.15$; maybe $M=0.43$; no $M=1.13$ ). As such, we considered the influence of these two factors separately when investigating their effects on the importance of age as a matching criterion.

For the analyses presented below, we included all users in our models but only present predictions for those with up to three children. This is because so few of our users reported having four or more children $(1.3 \%$; see Table $\mathrm{S} 1)$ that we chose not to make predictions based on this underrepresented sample of the population.

\section{Having children}

Using a robust linear regression (MASS package - Venables \& Ripley, 2002) with robust tests of significance (sfsmisc package - Maechler, 2020), we predicted the importance of age as a matching criterion. The three-way interaction between user age, gender, and the number of children that users had was statistically significant (see Table S2). The difficulty with analysing large datasets is that statistical significance is almost guaranteed due to the extremely small standard errors (Khalilzadeh \& Tasci, 2017; McFarland \& McFarland, 2015). As such, we explored the results visually and considered practical significance based on the predictions of the model. Figure 1 confirms our original prediction that users who already had children rated the importance of age as lower when finding a match. However, this effect decreased as user age increased. Interestingly, 
it is also clear that overall, women considered age as a matching criterion to be more important in comparison with men.

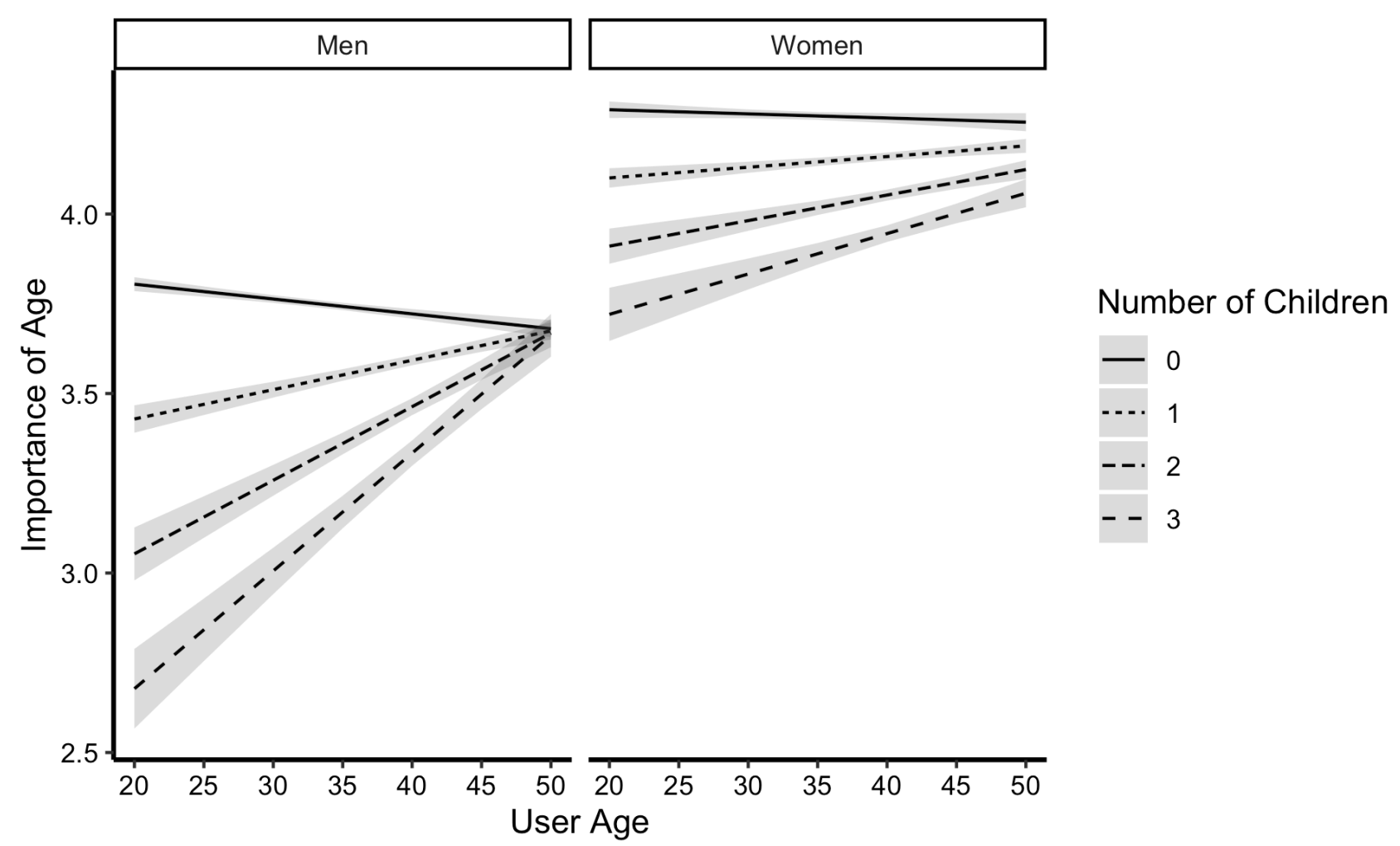

Figure 1. The effect of the number of children that users had when predicting the importance of age as a matching criterion from user age. Models are separately illustrated for male and female users. The grey regions represent $95 \%$ confidence bands.

The differences between those with and without children are further illustrated in Figure 2, where pairwise comparisons were carried out at five-year intervals for the predicted difference in the importance of age across the users' age range. Positive values represent a higher rated importance for those without children. For older users, rated importance showed little difference due to having children (values close to zero) for both genders. However, for the youngest men (aged 20), the predicted importance of age was rated more than 1 unit higher on the scale for those who did not have children in comparison with those who had three children. For the youngest 
women, we found smaller effects of having children (around half of the size seen in men) on rated importance.

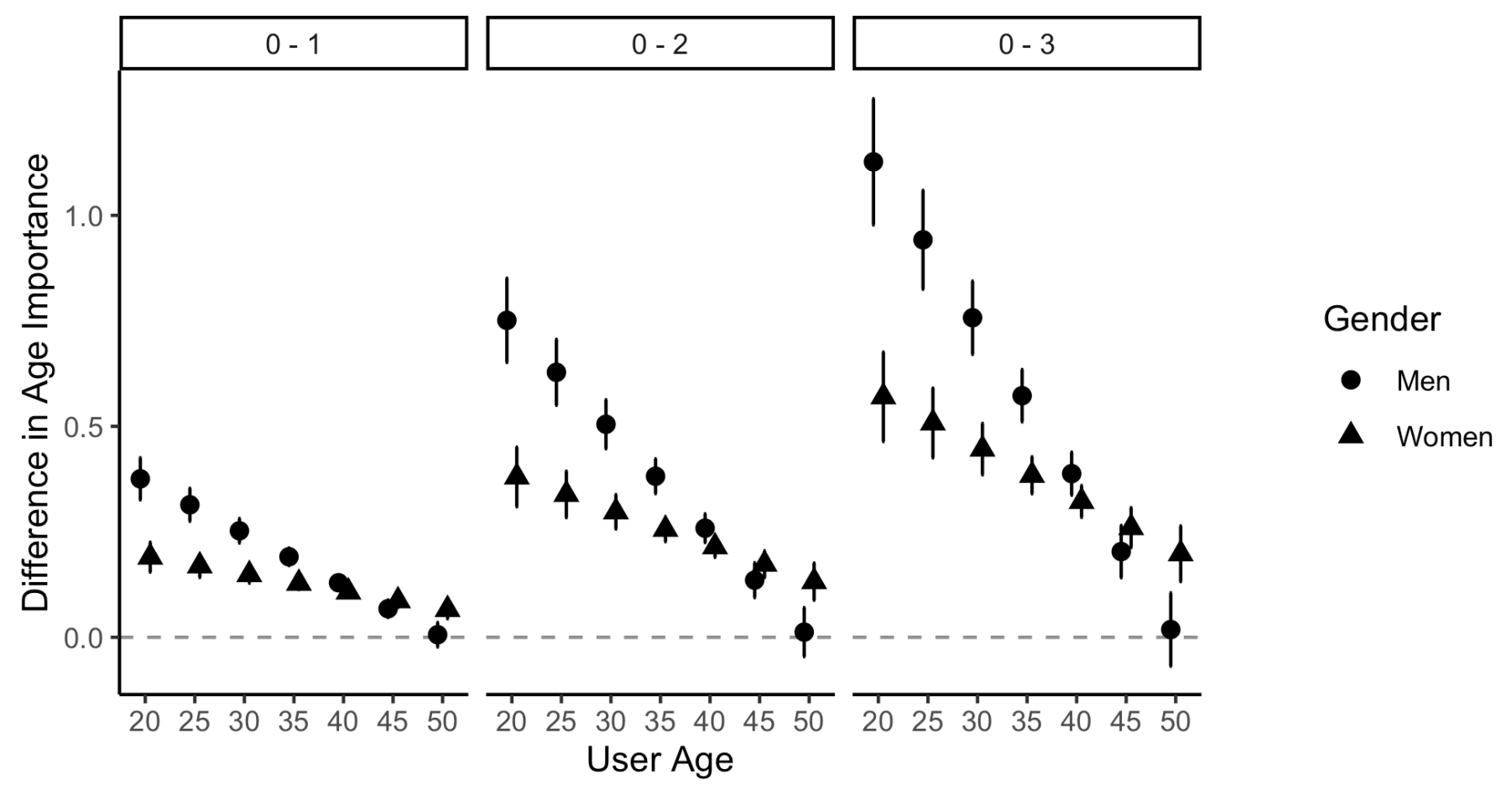

Figure 2. The difference in the importance of age between users with varying numbers of children. These differences are calculated as noted at the top of each panel. Error bars represent 95\% confidence intervals, with those overlapping zero (grey dashed reference line) demonstrating no difference due to having children.

\section{Wanting children}

Using a robust linear regression, we again predicted the importance of age as a matching criterion. The three-way interaction between user age, gender, and wanting children was statistically significant (see Table S3).

As before, we explored the results visually and considered practical significance. Figure 3 shows a larger difference in rated importance of age for older users when comparing those who did want children with the other two categories. However, even this difference only amounted to 
approximately 0.25 units on the scale. Female and male users demonstrated a similar pattern of results, although again, women considered age as a matching criterion to be more important in comparison with men.

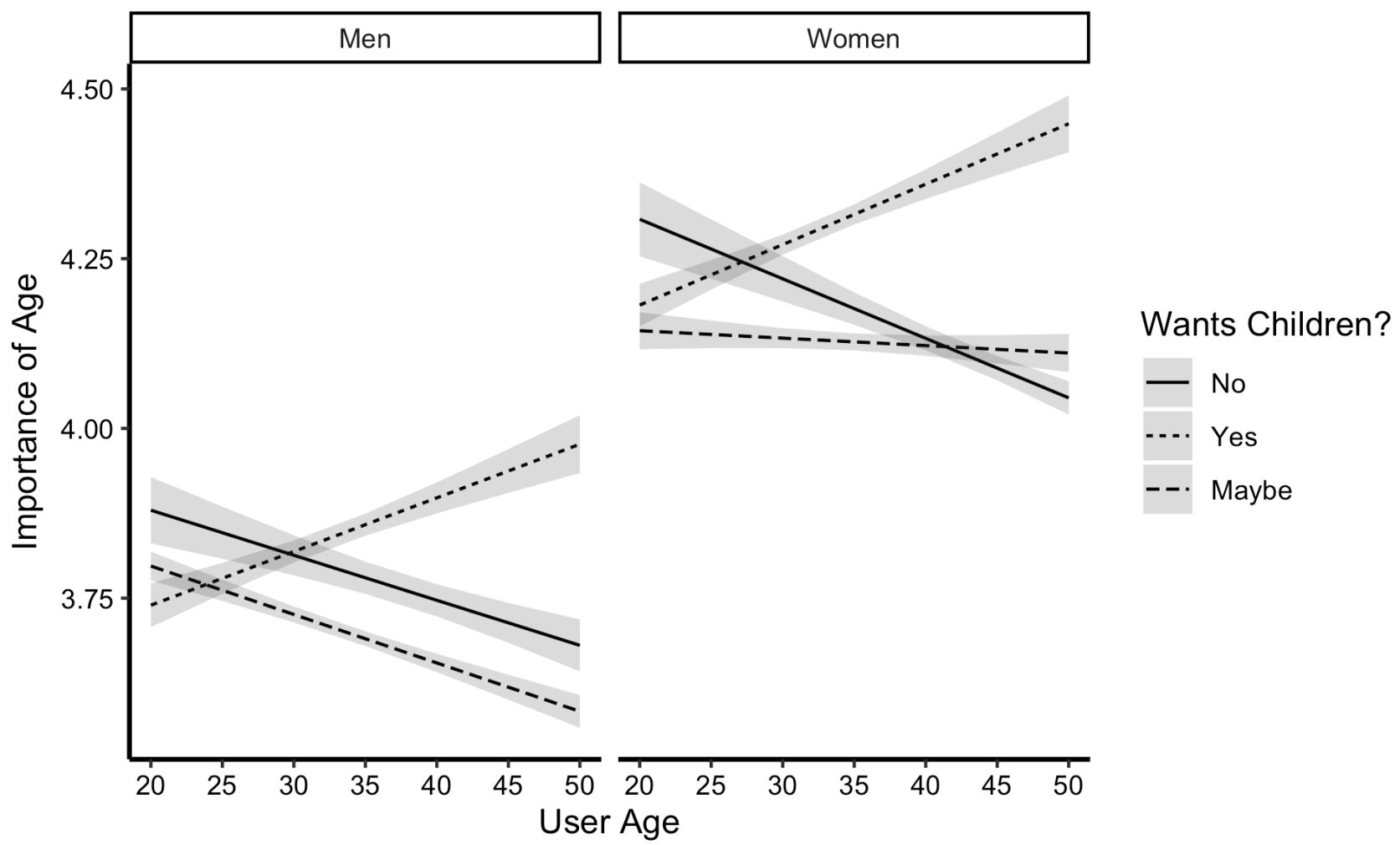

Figure 3. The effect of wanting (more) children when predicting the importance of age as a matching criterion from user age. Models are separately illustrated for male and female users. The grey regions represent $95 \%$ confidence bands.

These differences are further illustrated in Figure 4, where pairwise comparisons were carried out at five-year intervals for the predicted difference in age preferences across the users' age range. In all cases, as noted above, differences between categories were minimal in terms of scale units. 


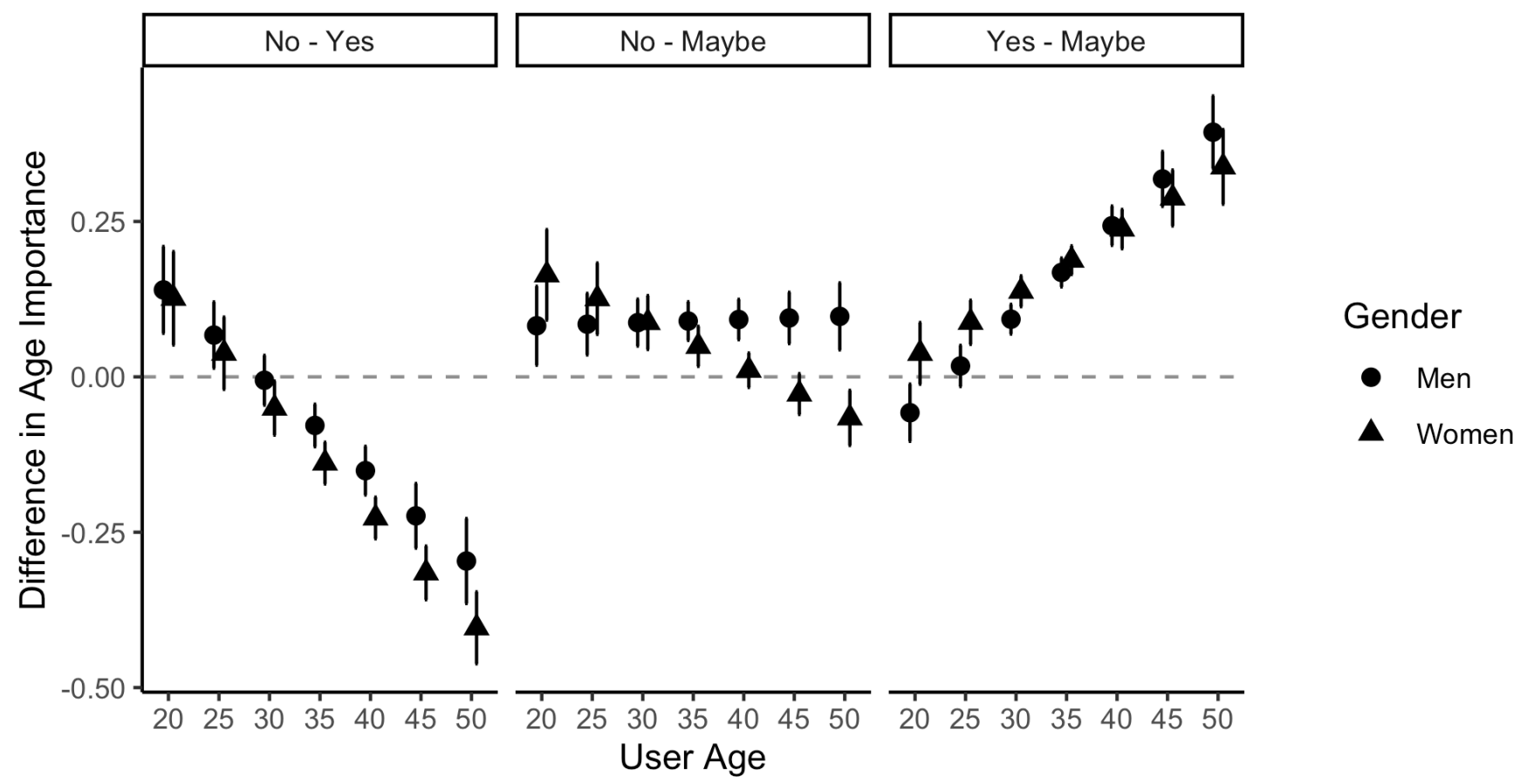

Figure 4. The difference in the importance of age between users in terms of whether they wanted (more) children. These differences are calculated as noted at the top of each panel. Error bars represent 95\% confidence intervals, with those overlapping zero (grey dashed reference line) demonstrating no difference due to category.

\section{Discussion}

In line with our predictions, users who had children rated age to be less important as a matching criterion. This result was more apparent for younger users, and men in particular. Regarding the differences due to wanting children, for older users, those who wanted children rated age as more important, while this pattern was reversed for younger users. However, in both cases, the effect was small in terms of scale units. Finally, in both analyses, women considered age to be more important in comparison with men.

\section{Study 2}


While Study 1 provided evidence that age was differentially important as a dating consideration for those with and without children (and, to a lesser extent, those who did and did not want children), these results could not inform regarding the direction of any age preferences. Here, we investigated whether having or wanting children predicted the preferred minimum and maximum age of those who users were willing to date.

\section{Method}

\section{Dataset}

Profile information was again obtained from eharmony UK. This new dataset comprised information taken from 1,043,554 website profiles which had been pre-identified by the company as valid (i.e., not fraudulent or scam users). There was no overlap between this sample and the user data analysed in Study 1. In all cases, users were not currently in a relationship. For each profile, the user had self-reported (upon registration) their age, sexual orientation, ethnicity, and gender. Importantly for the current study, users also reported the minimum and maximum age of prospective dating partners, the number of children they had, and whether they wished to have (more) children (yes/no preference/no).

From this initial sample, we chose to retain only heterosexual users as our hypotheses were based upon the association between having or wanting children and partners' age preferences from an evolutionary perspective. As before, we also excluded those users who reported having more than seven children since these responses were considered to be false. Regarding the minimum and maximum age of those who users wanted to date, the website provides default values (based on the user's age) that can be altered after registration has been completed. Given that we were unable to determine whether users chose to keep these default values or simply failed to specify their own, we also excluded all users where both the minimum and maximum values remained unchanged from 
the defaults. That is, users would have had to alter at least one of these values to be included in the dataset.

As in Study 1, we chose to include only users aged at least 20 years old in order to avoid potentially false information. In addition, users were unable to set their minimum age preference to below 18. As a result, many users might have been willing to date people younger than 18 but chose this value as the lowest available. By considering users with a minimum age of 20 , this meant that even the youngest members of our sample could demonstrate a (limited) willingness to date partners younger than themselves. Again, we also chose to include only users aged 50 and below.

The final sample comprised 486,382 users (46.26\% women; $61.12 \%$ White; age $M=33.31$ years, $S D=9.11$ years) from the following countries: US $-68.4 \%$, UK $-15.5 \%$, Australia $-9.6 \%$, Canada $-6.5 \%$.

\section{Statistics}

Due to the fact that users cannot specify preferred minimum or maximum ages younger than 18 , the difference between preferred and actual age was necessarily smaller for younger in comparison with older users. As such, we again tested for heteroskedasticity and found evidence of it for both maximum, $\chi^{2}(1)=25857, p<.001$, and minimum age preferences, $\chi^{2}(1)=27899, p<$ .001 . As in Study 1, we chose to report robust standard errors for all analyses, which adjust for heteroskedasticity, and fit our data using robust linear models.

Through inspection of both probability-probability and quantile-quantile plots, we observed deviations from multivariate normality in our data. However, as noted previously, a linear regression approach is still valid for large data sets (Lumley et al., 2002).

\section{Results}


As in Study 1, we found substantial overlap in the data when considering having children and wanting children (see Table S4). A one-way between subjects ANOVA found significant differences between groups (wanting children: yes/no preference/no) in terms of the number of children that users already had, $F(2,486379)=20787, p<.001, \eta^{2}=0.08$. In all cases, paired comparisons were statistically significant (all $p \mathrm{~s}<.001$; yes $M=0.18$; no preference $M=0.75$; no $M=1.71)$. As such, we considered the influence of these two factors separately when investigating their effects on age preferences.

Following the same reasoning as for Study 1, the analyses presented below included all users in our models but only present predictions for those with up to three children. Again, users who reported having four or more children (3.2\%; see Table S4) were underrepresented in our sample.

\section{Having children}

Using a robust linear regression, with robust tests of significance, we predicted the preferred minimum age of users when dating, with user age, gender, and number of children as predictors. We also repeated this process in order to predict the preferred maximum age of users, building a separate model for this outcome variable. In both cases, the three-way interaction between user age, gender, and number of children was statistically significant (see Tables S5 and S6).

As in Study 1, we explored the results visually and considered practical significance. Figure 5 shows the familiar pattern whereby women's preferred age range typically includes more men who are older rather than younger than themselves, while men show the opposite pattern of results (e.g., Antfolk et al., 2015). In addition, we see differences in users' preferences as a result of the number of children they had. 


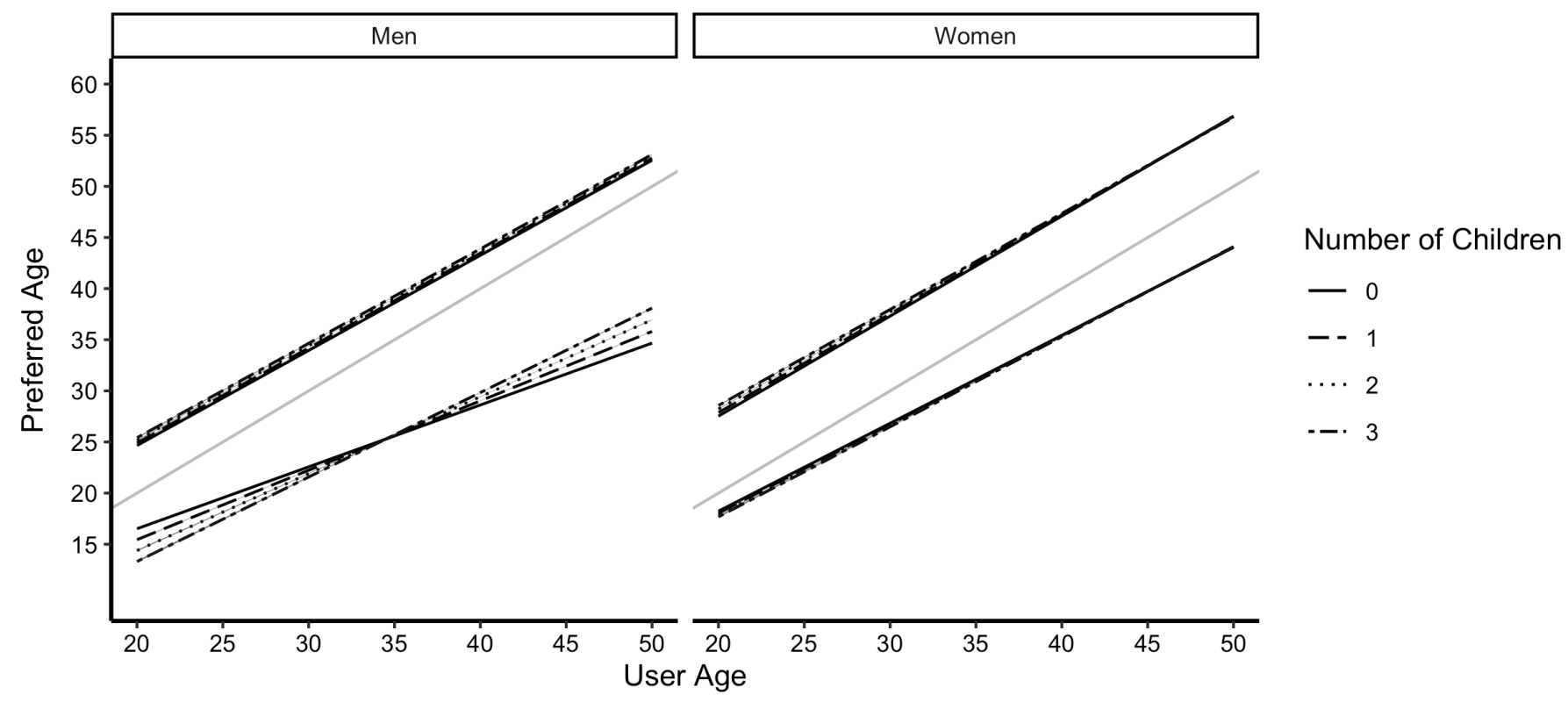

Figure 5. The effect of the number of children that users had when predicting preferred age from user age. Models are separately illustrated for male and female users. The grey line represents the reference line $(\mathrm{Y}=\mathrm{X})$. Lines appearing above this reference show predictions for the maximum preferred age, while lines below the reference show predictions for the minimum preferred age. Confidence bands are plotted but are not visible due to the large sample size.

These differences are further illustrated in Figure 6, where pairwise comparisons were carried out at five-year intervals for the predicted difference in age preferences across the users' age range. Positive values represent a higher age preference for those without children. While differences in the maximum age preference (Figure 6 lower) due to having children appear to have little practical significance (a change of between 0 and 1 year in preferred age), we see a more noticeable effect for the minimum age preferred by male users (Figure 6 upper). For 50-year-old men, those without children show a predicted minimum age preference that is more than 3 years lower than men of the same age who have three children. However, for 20 -year-old men, the pattern is reversed and those without children increase their minimum age by over 3 years when compared to those with three children. As with maximum preferred age, the effect of having children on women's minimum age preferences is of little practical significance. 

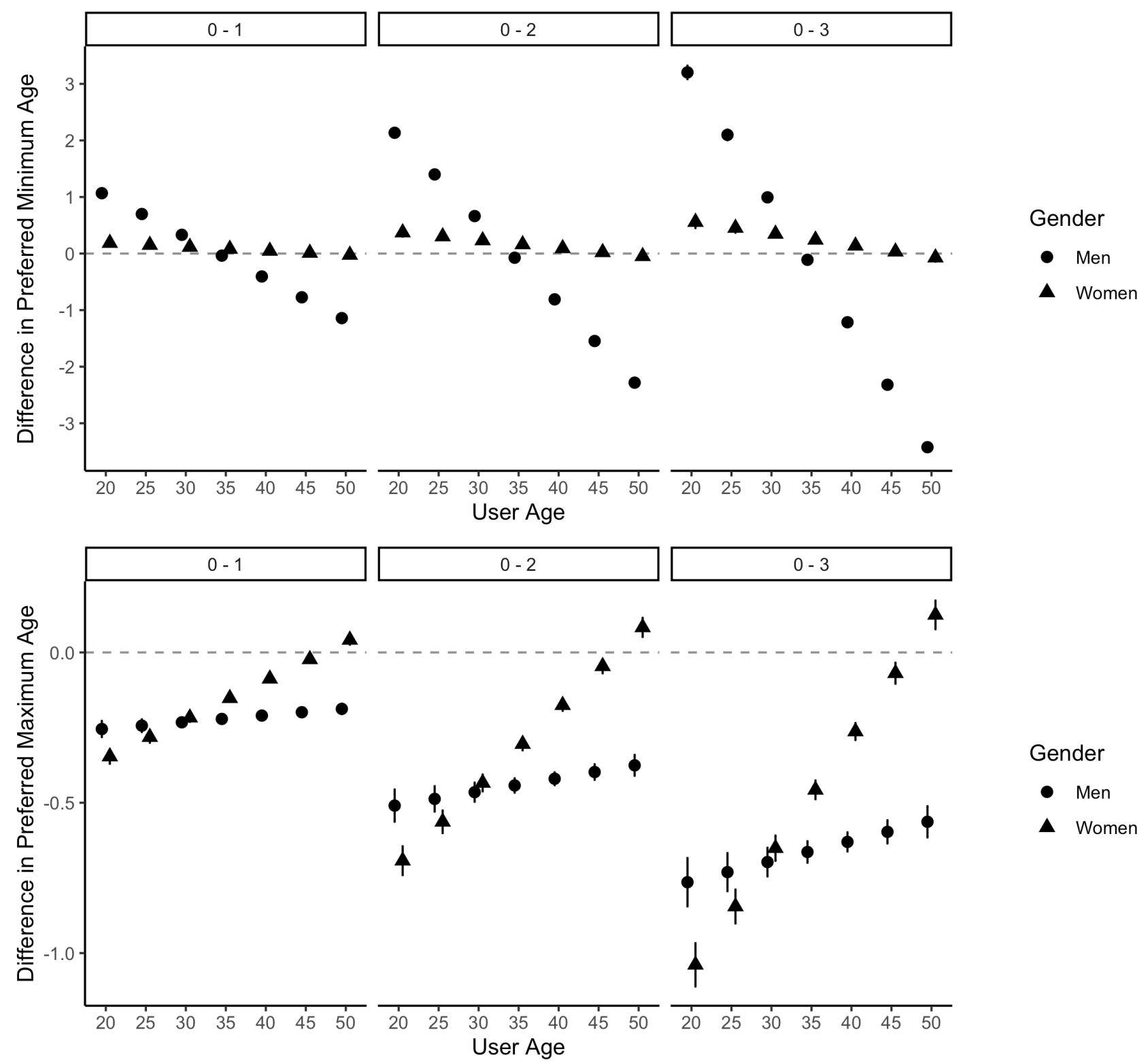

Figure 6. The difference in preferred age between users with varying numbers of children. These differences are illustrated for both preferred minimum (upper) and maximum age (lower), with differences calculate as noted at the top of each panel. Error bars represent $95 \%$ confidence intervals, with those overlapping zero (grey dashed reference line) demonstrating no difference due to having children.

It is important to note, however, that we should not place much confidence in model interpretation for these minimum age preferences for the youngest men. The model predicted preferred minimum ages below 18, which were impossible in reality, given the restrictions imposed on the user by the website. Most likely, this resulted from limitations within the dataset itself, 
whereby the youngest users (20 years old) could only provide a minimum response (18 years old) close to their own age, while the oldest users (50 years old) had far greater scope for their responses. Although the use of a robust linear regression addressed the issue of heteroskedasticity regarding the coefficients and standard errors, the problematic nature of these response limitations precludes interpretation at the lowest end of the user age range for the preferred minimum age.

\section{Wanting children}

Using a robust linear regression, we predicted the preferred minimum age of users when dating. We also repeated this process in order to predict the preferred maximum age of users, building a separate model for this outcome variable. In both cases, the three-way interaction between user age, gender, and wanting children was statistically significant (see Tables S7 and S8).

As before, we explored the results visually and considered practical significance. Figure 7 shows a similar pattern to Figure 5, whereby women's preferred age range typically includes more men who are older rather than younger than themselves, while men show the opposite pattern of results. In addition, we see differences in users' preferences as a result of their wanting children or not.

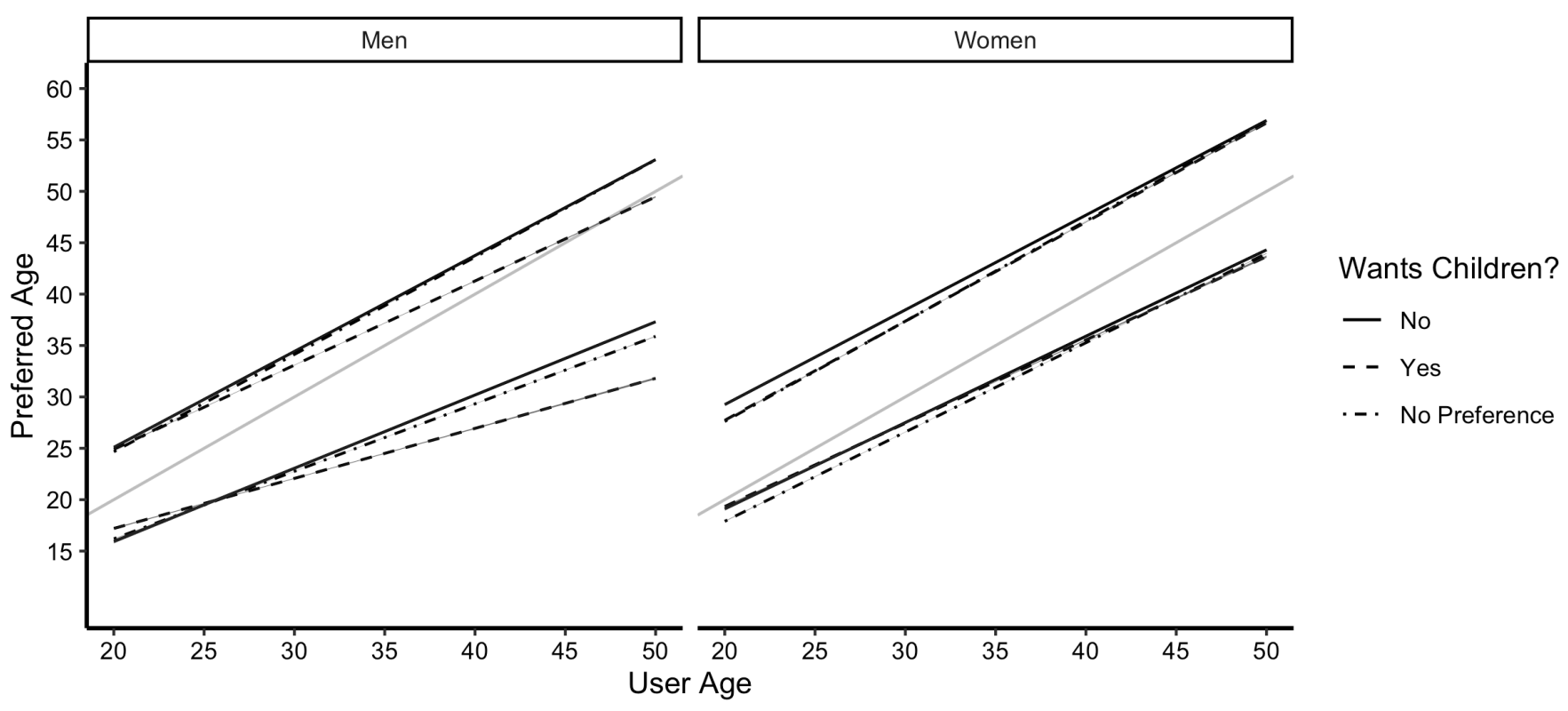


Figure 7. The effect of wanting (more) children when predicting preferred age from user age. Models are separately illustrated for male and female users. The grey line represents the reference line $(\mathrm{Y}=\mathrm{X})$. Lines appearing above this reference show predictions for the maximum preferred age, while lines below the reference show predictions for the minimum preferred age. Confidence bands are plotted but are not visible due to the large sample size.

These differences are further illustrated in Figure 8, where pairwise comparisons were carried out at five-year intervals for the predicted difference in age preferences across the users' age range. For the preferred minimum age (Figure 8 upper), we see little practical difference for female users. However, men who did not want children reported a substantially higher preferred minimum age preference as they got older, with 50-year-olds showing a predicted lower bound that was more than 5 years older when they did not want children in comparison with when they did. (A similar pattern was also found when comparing men with no preference to those who wanted children.) For the preferred maximum age (Figure 8 lower), women and men showed contrasting patterns. Older men who did not want children (or who expressed no preference) reported an upper bound that was more than 3 years higher (for 50-year-olds) than those who wanted children, while women of that age who did versus did not want children (or expressed no preference) showed little difference in their maximum age preference. However, younger women who did not want children reported an upper bound that was around 1.5 years higher (for 20-year-olds) in comparison with those who wanted children or expressed no preference. 


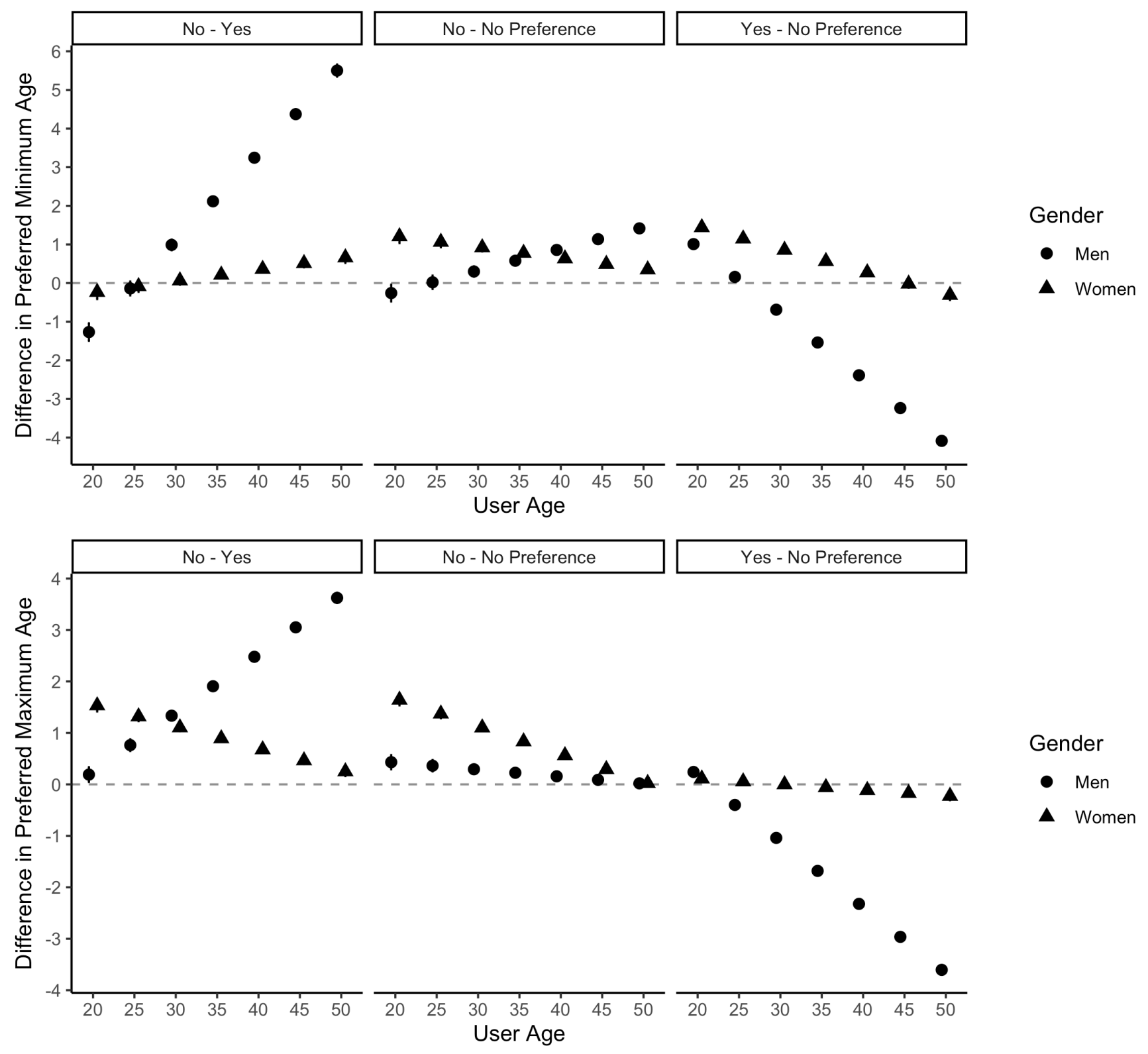

Figure 8. The difference in preferred age between users in terms of whether they wanted (more) children. These differences are illustrated for both preferred minimum (upper) and maximum age (lower), with differences calculate as noted at the top of each panel. Error bars represent 95\% confidence intervals, with those overlapping zero (grey dashed reference line) demonstrating no difference due to wanting children.

As with having children, above, we were precluded from interpreting model predictions for the preferred minimum age for the lowest end of the user age range due to response limitations within the dataset. 


\section{Discussion}

Having children had little practical effect on women's age preferences. However, older men without children demonstrated a lower minimum preferred age when compared to those with children. These results were mirrored for wanting children (with 'wanting' being equivalent to 'not having'). For the preferred maximum age, older men who did not want children reported higher values than those who wanted children. Interestingly, this same pattern was found for younger women.

\section{General Discussion}

In line with evolutionary theories, previous research has demonstrated that sex differences in mating strategies are apparent in age preferences when dating. Men have been shown to prefer women in their early to mid-twenties irrespective of their own age, while women express a preference for men who are older than themselves but not significantly so (e.g., Antfolk et al., 2015). Indeed, the general findings of Study 2 replicated these results. However, given that the underlying (ultimate) explanation for these patterns relies on the increase in inclusive fitness, it follows that having or wanting children should affect such outcomes. In two large samples of online daters, we addressed this prediction.

When reporting how important age was as a matching criterion (Study 1), users who had children gave lower ratings than those who did not. Practically, this amounted to approximately a one-unit difference on a 1-7 response scale for the youngest men, and a decrease in ratings with an increase in the number of children that users had. Women also showed statistically significant effects (although small in practical terms) due to having children, while it was clear that, in general, women considered age to be more important as a matching criterion in comparison with men. 
Exploration of whether users wanted children or not revealed little of practical significance (i.e., response scale units), perhaps suggesting that this had less of a relationship with the perceived importance of age in comparison with having children.

Men in their twenties may place more importance on the age of a partner if they do not already have children because female youth and fertility provide the greatest potential for reproduction (Dunson et al., 2002; Wood, 1989). For men in their forties, the likelihood of wanting (more) children decreases, no matter whether they have children or not, and as a result, the age of a potential partner becomes less important.

By exploring minimum and maximum age preferences (Study 2), we were able to investigate the real-world restrictions that users placed upon their dating behaviours, since such preferences have a direct and limiting effect on who users can be matched with. We found that older men without children selected a minimum age that was up to four years lower than men of the same age who have children, with this difference being larger when comparing with users who have more children. This can be explained by the desire to maximise reproductive potential in a partner through having more time together before the onset of menopause, and because younger women are significantly more likely to have healthier pregnancies (e.g., van Noord-Zaadstra et al., 1991).

For the most part, this pattern was also present when investigating wanting children, where older men who did want children reported a minimum that was more than five years lower than men of the same age who did not. In conjunction with this, older men who wanted children also reported a lower maximum age by over three years in comparison with those who did not, again limiting their preferred range to women more suited to reproduction. Indeed, these men excluded women who were their age or older.

For women, the only effect of practical significance was found in relation to wanting children. Younger women who wanted children reported a lower maximum age by 1.5 years in comparison with those who did not. This may reflect the desire to find men who have good resource potential while also being more compatible (e.g., being closer to their own age; Kenrick \& Keefe, 1992) or it 
may simply be a dating strategy (as men get older, women might assume they already have children and/or do not desire more). The explanation for this result therefore remains to be established.

It is important to consider these results within the framework of ultimate and proximate explanations introduced earlier. At the ultimate level, any 'function' is a result of the relative gain in our inclusive fitness. In terms of proximate mechanisms, our attraction to certain age-related features may be altered as a result of having or wanting children, which in turn, influences our dating behaviours. While the ultimate goal is reproduction (and, therefore, increased inclusive fitness), the proximate mechanism is the desire to copulate (Pérusse, 1993). For our ancestors, copulation and reproduction were essentially equivalent (without any effective means of contraception), which meant that there was no disadvantage to our evolving a desire for the former rather than the latter. Of course, these two outcomes are no longer inseparable, but our results demonstrate that those who we desire to date reflect whether we have or want children.

Although we might think of having or wanting children as factors that affect subsequent mating decisions, it is unlikely, for the most part, that these reflect conscious decisions that directly alter mate choices. It is certainly possible that, on occasion, daters might explicitly select a potential mate because they themselves want more children, and that person represents a good option. However, such decisions will typically be more implicit. While daters are usually aware of their desire to reproduce when questioned, we are not arguing that such desires were consulted directly when daters laid out their age preferences online.

While the age of considered dating partners may be independent of age preferences expressed by the opposite sex, real-world behaviours and outcomes will likely reflect a compromise (Antfolk, 2017). For example, daters may face a trade-off between inclusivity (e.g., men, in particular, avoiding the exclusion of women from their initial dating pool by mostly 'swiping right' in Tinder; Tyson et al., 2016) and impression formation (e.g., not wanting to appear too inclusive and, as a result, unappealing to the opposite sex by having a broad age range for their preference). Here, daters using eharmony UK specified their age preferences but these were not published on their 
profiles for others to view. As such, age preferences served to limit the users that someone could be matched with but did not, of themselves, affect the impressions formed by those users. Therefore, we investigated age preferences in the present work that more closely mirrored potential partners that users would consider dating, although these had real-world effects in terms of whom users could actually date.

The current study considered online dating profiles provided by eharmony UK. Do these daters' preferences reflect mate selection more generally, and the choices made by the population as a whole? First, as of 2017, around $40 \%$ of heterosexual couples in the US report meeting online, according to recent research (Rosenfeld et al., 2019). As such, it seems likely that online daters are indeed representative of the general population. Second, in terms of their preferences, eharmony users tend to be interested in forming serious, long-term relationships (Long, 2010). While evidence suggests that mate preferences may not substantially differ across short- and long-term relationships (Hitsch et al., 2010), it is also likely that both men and women are more "selective" when choosing long-term partners (Sprecher et al., 1994). Given that the current work focussed on whether daters wanted (more) children or not, it makes sense to consider long-term dating preferences where this would be of more relevance. However, future research might investigate the relationship between wanting or having children and age preferences in short-term dating behaviours.

Although we investigated modern, online dating behaviours in the current work, it is clear that this setting is notably different from the environment within which we adapted. As such, one might question how our findings speak to mate selection more broadly. While humans have not adapted to find mates via the internet, it seems likely that such forums were designed to match at least some of our psychological adaptations. For example, dating websites typically allow users to specify their preferred age range precisely because age is something we have adapted to consider as important. Therefore, although the mating context is certainly different from those within which we have evolved, we can reasonably argue that this current setting has been designed to cater to those key features that reflect past environments. 
To conclude, we argued that evolutionary theory predicts differing desires regarding mate selection when comparing those who have (or do not want) children with those who do not have (or want) children. These predictions were borne out in two large samples of online daters, supporting the potential influence (given the correlative nature of the data) of having or wanting children on male dating behaviours. Predictions were less clear regarding women, where the preference for younger or older men is more nuanced, and indeed, we found little of practical significance in how these factors predicted their responses. Taken together, our findings extend research on age preferences to incorporate these theoretically significant, yet previously unexplored, components of mate selection.

\section{Supplemental Material}

The supplemental material is available in the online version of the article.

\section{References}

Antfolk, J. (2017). Age limits: Men's and women's youngest and oldest considered and actual sex partners. Evolutionary Psychology, 15(1), 1-9.

Antfolk, J., Salo, B., Alanko, K., Bergen, E., Corander, J., Sandnabba, N. K., \& Santtila, P. (2015). Women's and men's sexual preferences and activities with respect to the partner's age: Evidence for female choice. Evolution and Human Behavior, 36(1), 73-79.

Apter, D. (1980). Serum steroids and pituitary hormones in female puberty: A partly longitudinal study. Clinical Endocrinology, 12(2), 107-120.

Breusch, T. S., \& Pagan, A. R. (1979). A simple test for heteroscedasticity and random coefficient variation. Econometrica, 47(5), 1287-1294. 
Buss, D. M. (1989). Sex differences in human mate preferences: Evolutionary hypotheses tested in 37 cultures. Behavioral and Brain Sciences, 12(1), 1-14.

Buss, D. M., \& Schmitt, D. P. (1993). Sexual strategies theory: An evolutionary perspective on human mating. Psychological Review, 100(2), 204-232.

Buss, D. M., \& Schmitt, D. P. (2019). Mate preferences and their behavioral manifestations. Annual Review of Psychology, 70, 77-110.

Buss, D. M., Shackelford, T. K., \& LeBlanc, G. J. (2000). Number of children desired and preferred spousal age difference: Context-specific mate preference patterns across 37 cultures. Evolution and Human Behavior, 21(5), 323-331.

Buunk, B. P., Dijkstra, P., Kenrick, D. T., \& Warntjes, A. (2001). Age preferences for mates as related to gender, own age, and involvement level. Evolution and Human Behavior, 22(4), 241-250.

Conroy-Beam, D., \& Buss, D. M. (2019). Why is age so important in human mating? Evolved age preferences and their influences on multiple mating behaviors. Evolutionary Behavioral Sciences, 13(2), 127-157.

Conway, J. R., Noë, N., Stulp, G., \& Pollet, T. V. (2015). Finding your Soulmate: Homosexual and heterosexual age preferences in online dating. Personal Relationships, 22(4), 666-678.

Daly, M., \& Wilson, M. (1983). Sex, evolution, and behavior. Boston: Willard Grant Press.

Dinh, R., Gildersleve, P., \& Yasseri, T. (2018). Computational courtship: Understanding the evolution of online dating through large-scale data analysis. arXiv. https://arxiv.org/abs/1809.10032

Dunn, M. J., Brinton, S., \& Clark, L. (2010). Universal sex differences in online advertisers age preferences: Comparing data from 14 cultures and 2 religious groups. Evolution and Human Behavior, 31(6), 383-393.

Dunson, D. B., Colombo, B., \& Baird, D. D. (2002). Changes with age in the level and duration of fertility in the menstrual cycle. Human Reproduction, 17(5), 1399-1403. 
Gruhn, J. R., Zielinska, A. P., Shukla, V., Blanshard, R., Capalbo, A., Cimadomo, D., Nikiforov, D., Chan, A. C.-H., Newnham, L. J., Vogel, I., Scarica, C., Krapchev, M., Taylor, D., Kristensen, S. G., Cheng, J., Ernst, E., Bjørn, A.-M. B., Colmorn, L. B., Blayney, M., ... Hoffmann, E. R. (2019). Chromosome errors in human eggs shape natural fertility over reproductive life span. Science, 365(6460), 1466-1469.

Gustavsson, L., Johnsson, J. I., \& Uller, T. (2008). Mixed support for sexual selection theories of mate preferences in the Swedish population. Evolutionary Psychology, 6(4), 575-585.

Hawkes, K., \& Smith, K. R. (2010). Do women stop early? Similarities in fertility decline in humans and chimpanzees. Annals of the New York Academy of Sciences, 1204, 43-53.

Hitsch, G. J., Hortaçsu, A., \& Ariely, D. (2010). Matching and sorting in online dating. American Economic Review, 100(1), 130-63.

Kenrick, D. T., \& Keefe, R. C. (1992). Age preferences in mates reflect sex differences in human reproductive strategies. Behavioral and Brain Sciences, 15(1), 75-91.

Khalilzadeh, J., \& Tasci, A. D. A. (2017). Large sample size, significance level, and the effect size:

Solutions to perils of using big data for academic research. Tourism Management, 62, 89-96.

Long, B. L. (2010). Scripts for online dating: A model and theory of online romantic relationship initiation [Unpublished doctoral dissertation]. Bowling Green State University, $\mathrm{OH}$.

Lumley, T., Diehr, P., Emerson, S., \& Chen, L. (2002). The importance of the normality assumption in large public health data sets. Annual Review of Public Health, 23(1), 151-169.

Maechler, M. (2020). sfsmisc: Utilities from ‘Seminar fuer Statistik’ ETH Zurich. R package version 1.1-7. https://CRAN.R-project.org/package=sfsmisc

Marshall, W. A., \& Tanner, J. M. (1969). Variations in pattern of pubertal changes for girls. Archives of Disease in Childhood, 44(235), 291-303.

Marshall, W. A., \& Tanner, J. M. (1970). Variations in the pattern of pubertal changes in boys. Archives of Disease in Childhood, 45(239), 13-23.

Mayr, E. (1963). Animal species and evolution. Harvard University Press. 
McDowell, M. A., Brody, D. J., \& Hughes, J. P. (2007). Has age at menarche changed? Results from the National Health and Nutrition Examination Survey (NHANES) 1999-2004. Journal of Adolescent Health, 40(3), 227-231.

McFarland, D. A., \& McFarland, H. R. (2015). Big data and the danger of being precisely inaccurate. Big Data \& Society, 2(2), 1-4.

National Health Service (2021, August 29). Menopause. https://www.nhs.uk/conditions/menopause

Pérusse, D. (1993). Cultural and reproductive success in industrial societies: Testing the relationship at the proximate and ultimate levels. Behavioral and Brain Sciences, 16, 267-322.

Phua, V. C., Sosa, C. J., \& Aloisi, K. (2018). Males prefer younger females: Age preference among online daters in the Dominican Republic. Sexuality \& Culture, 22(1), 39-47.

Rosenfeld, M. J., Thomas, R. J., \& Hausen, S. (2019). Disintermediating your friends: How online dating in the United States displaces other ways of meeting. Proceedings of the National Academy of Sciences, 116(36), 17753-17758.

Skopek, J., Schmitz, A., \& Blossfeld, H. -P. (2011). The gendered dynamics of age preferences Empirical evidence from online dating. Journal of Family Research, 23(3), 267-290.

Sohn, K. (2016). Men's revealed preferences regarding women's ages: Evidence from prostitution. Evolution and Human Behavior, 37(4), 272-280.

Sohn, K. (2017). Men's revealed preference for their mates' ages. Evolution and Human Behavior, $38(1), 58-62$.

Sprecher, S., Sullivan, Q., \& Hatfield, E. (1994). Mate selection preferences: Gender differences examined in a national sample. Journal of Personality and Social Psychology, 66(6), 10741080.

Tinbergen, N. (1963). On the aims and methods of ethology. Zeitschrift für Tierpsychologie, 20, 410-433.

Trivers, R. (1972). Parental investment and sexual selection. In B. Campbell (Ed.), Sexual selection and the descent of man (pp. 136-179). Chicago, IL: Aldine-Atherton. 
Tyson, G., Perta, V. C., Haddadi, H., \& Seto, M. C. (2016, August). A first look at user activity on tinder. In IEEE/ACM International Conference on Advances in Social Networks Analysis and Mining (pp. 461-466). IEEE.

van Noord-Zaadstra, B. M., Looman, C. W., Alsbach, H., Habbema, J. D., te Velde, E. R., \& Karbaat, J. (1991). Delaying childbearing: Effect of age on fecundity and outcome of pregnancy. British Medical Journal, 302, 1361-1365.

Venables, W. N., \& Ripley, B. D. (2002). Modern applied statistics with S (4th ed.). Springer.

Waynforth, D., \& Dunbar, R. I. M. (1995). Conditional mate choice strategies in humans: Evidence from 'Lonely Hearts' advertisements. Behaviour, 132(9), 755-779.

Wood, J. W. (1989). Fecundity and natural fertility in humans. Oxford Reviews of Reproductive Biology, 11, 61-109. 